\title{
Pre-hospital plasma transfusion: a valuable coagulation support or an expensive fluid therapy?
}

\author{
Christian Fenger-Eriksen ${ }^{1}$, Dietmar Fries ${ }^{2}$, Jean-Stephane David ${ }^{3}$, Pierre Bouzatt ${ }^{4}$ Marcus Daniel Lance ${ }^{5}$, \\ Oliver Grottke ${ }^{6}$, Donat R. Spahn ${ }^{7}$, Herbert Schoechl ${ }^{8,9}$ and Marc Maegele ${ }^{10^{*}}$
}

Two recent clinical trials with conflicting results have refuelled the discussion on pre-hospital plasma in trauma. The multicentre, cluster-randomized PAMPer trial assessed the efficacy and safety of two units of pre-hospital plasma versus standard care without plasma in 501 trauma patients at risk for haemorrhagic shock during air medical transport to a designated US trauma centre [1]. The mortality at 30 days was lower in the plasma compared to the standard care group $(23 \%$ vs $33 \% ; p=0.03)$. The randomized, placebo-controlled COMBAT trial compared the same plasma volume versus isotonic saline in 144 haemorrhagic shocked trauma patients within a US ground EMS and a single US trauma centre but mortality at 28 days did not differ between trial groups (15\% vs $10 \%$; n.s.) [2]. Table 1 summarizes the basic characteristics of both trials. The results from both trials need to be viewed with caution against their limitations and may not be translated directly into routine without addressing a number of critical issues.

A single drop in blood pressure as an inclusion criterion for both trials is problematic as pre-hospital hypotensive episodes can have non-bleeding reasons (e.g. anaesthesia, cardiac, spinal trauma or wrong readings), and, in PAMPer, half of the patients had received pre-hospital intubation/mechanical ventilation while for COMBAT no details were provided. Both trials aimed for patients "at risk for haemorrhagic shock" or "thought to be due to acute blood loss" but no signs of bleeding were considered for inclusion. Notably, 111 patients in PAMPer had received unspecified pre-treatment prior to inclusion which may have introduced bias. The time span for inclusion expanded over 3 years with trauma care subject to change over time, e.g. the increasing widespread use of antifibrinolytic tranexamic acid (TXA). In COMBAT, 10\%

\footnotetext{
* Correspondence: Marc.Maegele@t-online.de

${ }^{10}$ Department of Traumatology and Orthopedic Surgery, Cologne-Merheim Medical Center, Institute for Research in Operative Medicine (IFOM),

University Witten-Herdecke, Cologne, Germany

Full list of author information is available at the end of the article
}

of patients had received TXA while its use was not reported for PAMPer.

There was high mortality difference at 24-h and 28/ 30-days within both control arms which had assumingly received comparable US standard trauma care after hospital admission (Table 1). With identical entry criteria, this difference may only be explained by differences in injury severity, volume status and further pattern and/or patient care; but no specific details were provided. The comparison of injury severity between both trials is difficult due to different scores applied. However, the mortality in PAMPer was higher than in COMBAT and that reported elsewhere which limits the external validity of findings. In the European RETIC trial on early coagulation factor concentrates versus FFP in trauma the 30-day mortality was only $7.4 \%$ despite an ISS of 34 [3]. The German Trauma Registry (TR-DGU) confirms a mortality $<10 \%$ for an ISS 20-23 [4]. In PAMPer, there was no clinical benefit for plasma on the sequalae of hypovolaemichaemorrhagic shock as $32 \%$ versus $29 \%$ of patients died in haemorrhagic shock.

The underlying mechanism by which the two units of pre-hospital plasma may have promoted lower mortality in PAMPer remains speculative. In both trials, no relevant improvements in standard/viscoelastic coagulation assays were reported after pre-hospital plasma. A statistically relevant but clinically insignificant shorter prothrombin ratio was reported for the plasma group (1.2 vs 1.3) but cannot account for the observed difference in mortality. In COMBAT, more patients in the plasma group had an INR > 1.3. The INR quantifies only pro-coagulants and does not mirror concentrations of inhibitors. In trauma, INR can be prolonged despite upregulated thrombin generation potential [5]. Moreover, the INR of FFP is 1.3 [6]. Any beneficial effect of plasma to correct slightly elevated INR is futile and plasma has primarily an effect on coagulation parameters with extended volumes and

(c) The Author(s). 2019 Open Access This article is distributed under the terms of the Creative Commons Attribution 4.0 International License (http://creativecommons.org/licenses/by/4.0/), which permits unrestricted use, distribution, and reproduction in any medium, provided you give appropriate credit to the original author(s) and the source, provide a link to the Creative Commons license, and indicate if changes were made. The Creative Commons Public Domain Dedication waiver (http://creativecommons.org/publicdomain/zero/1.0/) applies to the data made available in this article, unless otherwise stated. 


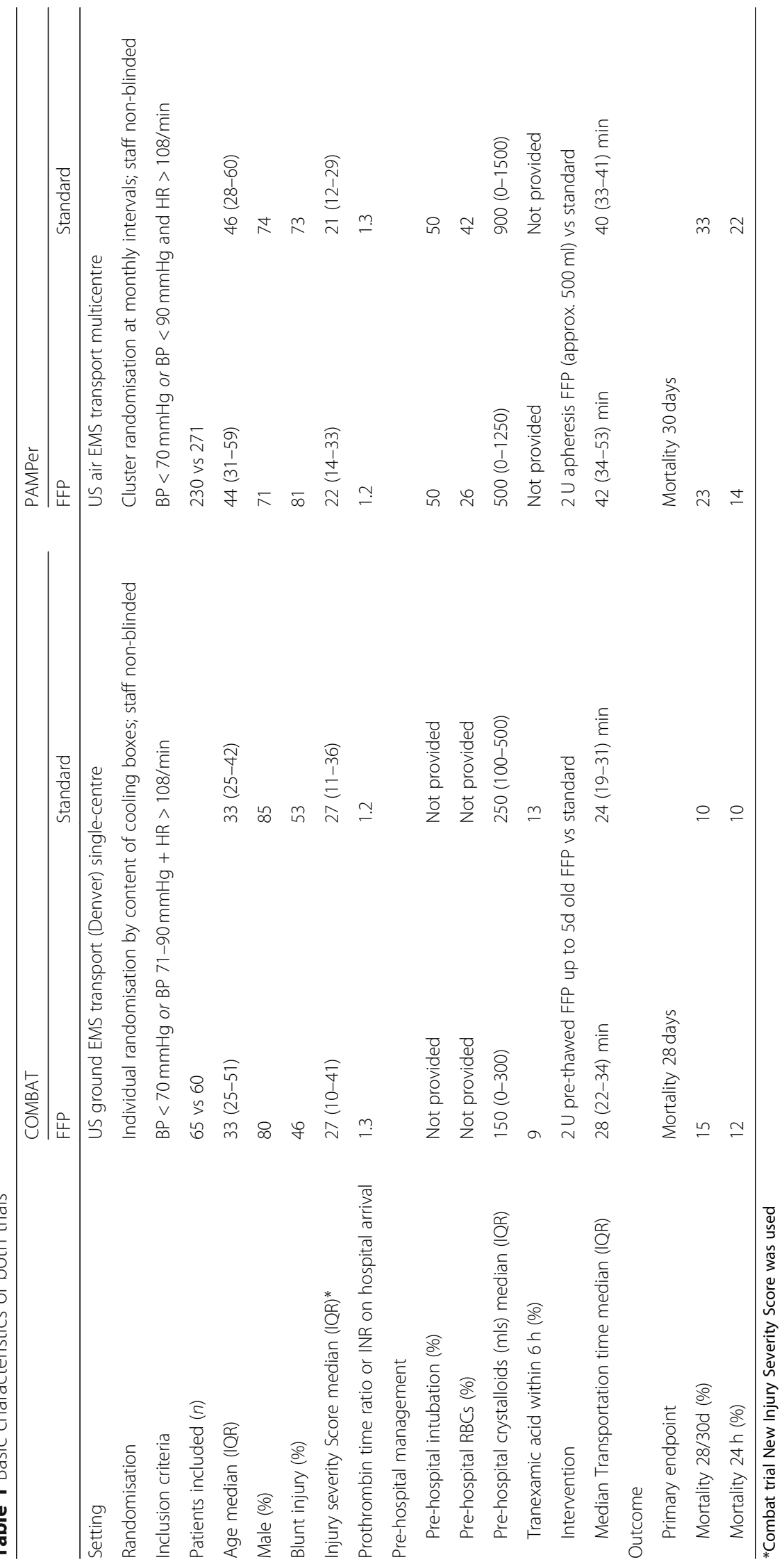


performs best in patients bleeding and coagulopathic. The negative effects of plasma need to be considered [7]; in COMBAT a trend towards higher MOF was observed in the plasma group ( $6 \%$ vs $2 \%$ ).

A large proportion of patients did not receive massive transfusion and most arrived at the hospital with the absence of clinical/laboratory signs of relevant coagulopathy. Furthermore, the number of pRBCs transfused in both trials was low and without any statistical difference. Notably, half of the PAMPer patients had received prehospital pRBCs. Moreover, no difference in mortality in massively transfused patients independent of pre-hospital plasma administration was reported. Accordingly, only patients with less severe injury would have benefited from pre-hospital plasma. In PAMPer, $42 \%$ of the standard care patients had received pre-hospital pRBCs (26\% in the FFP group) and almost twice as much crystalloids prior to hospital admission. Both measures could have contributed to the higher mortality in the standard care group in PAMPer. The transfusion trigger, however, remains unknown. For COMBAT, the authors admitted that median fibrinogen levels and other coagulation factors on hospital arrival were within reference ranges; and slightly higher in the standard of care non-FFP group.

As demonstrated, the pre-hospital administration of plasma to trauma patients is technically/logistically feasible both in air and ground EMS. However, the different conclusions of the trials leave the question unsolved to whether pre-hospital plasma may be of any clinical benefit for the target population. As PAMPer patients had received prehospital pRBCs and more intravenous fluids a simple "volume" effect cannot be excluded. Risk-benefits need to be balanced against other challenges, e.g. infrastructure, logistics and costs, and an early goal-directed approach using coagulation factor concentrates (e.g. fibrinogen, which is critically depleted first during bleeding [8]), TXA, and permissive hypotension along with surgical bleeding control to limit further blood loss and stabilize coagulation function pre-hospital may be an alternative as outlined in the updated European trauma guideline [9]. Protective effects to the glycocalyx and endothelial barrier integrity have been linked to the fibrinogen component rather than to plasma per se [10]. A median $3.8 \mathrm{~g}$ fibrinogen concentrate can increase clot stability by $5.2 \mathrm{~mm}$ at $5 \mathrm{~min}$ of viscoelastic test initiation while TXA can decrease lysis by $5.4 \%$ [11].

From a European perspective, the blind pre-hospital administration of plasma to potentially non-coagulopathic patients with short transportation times cannot be justified. More clearly defined studies are necessary to justify logistics and costs associated with pre-hospital blood product transfusion.

\section{Abbreviations}

ARDS: Acute respiratory distress syndrome; bpm: Beats per minute;

COMBAT: Plasma-first Resuscitation to Treat Haemorrhagic Shock during
Emergency Ground Transportation in an Urban Area: A Randomised Trial; CPP: Cerebral perfusion pressure; CRASH-2: Clinical Randomization of an Antifibrinolytic in Significant Hemorrhage 2 Trial; EMS: Emergency Medical Service; FFP: Fresh frozen plasma; INR: International Normalized Ratio; ISS: Injury Severity Score; MOF: Multiorgan failure; n.s.: Non-significant; PAMPer: Prehospital Plasma during Air Medical Transport in Trauma Patients at Risk for Haemorrhagic Shock Trial; pRBC: Packed red blood cells; PTr: Prothrombin ratio; RETIC: Reversal of Trauma-induced Coagulopathy using First-line Coagulation Factor Concentrates or Fresh Frozen Plasma Study; TBI: Traumatic brain injury; TR-DGU: TraumaRegistry of the Deutsche Gesellschaft für Unfallchirurgie; TXA: Tranexamic acid; US: United States

\section{Acknowledgements}

None.

\section{Authors' contributions}

CFE, DF, JSD, PB, MDL, OG, DRS, HS and MM conceived the idea for the manuscript. The first draft was written by CFE and MM. All authors contributed to and approved the final version.

\section{Funding}

This study received no funding. Publication costs were funded by the authors.

Availability of data and materials

Not applicable.

Ethics approval and consent to participate

Not applicable.

Consent for publication

Not applicable.

\section{Competing interests}

CFE received travel support, lecture and advisory board fees and research support from CSL Behring, TEM International/IL-Werfen and LFB Biomedicaments. DRS received honoraria or travel support for consulting or lecturing from Danube University of Krems, Austria, US Department of Defense, Washington, USA, European Society of Anesthesiology, Brussels, BE, Korean Society for Patient Blood Management, Seoul, Korea, Korean Society of Anesthesiologists, Seoul, Korea, Baxter AG, Volketswil, Switzerland, Baxter S.p.A., Roma, Italy, Bayer AG, Zürich, Switzerland, Bayer Pharma AG, Berlin, Germany, B. Braun Melsungen AG, Melsungen, Germany, Boehringer Ingelheim GmbH, Basel, Switzerland, Bristol-Myers-Squibb, Rueil-Malmaison Cedex, France and Baar, Switzerland, CSL Behring GmbH, Hattersheim am Main, Germany and Berne, Switzerland, Celgene International II Sàrl, Couvet, Switzerland, Curacyte AG, Munich, Germany, Daiichi Sankyo AG, Thalwil, Switzerland, GlaxoSmithKline GmbH \& Co. KG, Hamburg, Germany, Haemonetics, Braintree, MA, USA, Instrumentation Laboratory (IL-Werfen), Bedford, MA, USA, LFB Biomédicaments, Courtaboeuf Cedex, France, Merck Sharp \& Dohme, Kenilworth, New Jersey, USA,

Octapharma AG, Lachen, Switzerland, Organon AG, Pfäffikon/SZ, Switzerland, PAION Deutschland GmbH, Aachen, Germany, Pharmacosmos A/S, Holbaek, Denmark, Photonics Healthcare B.V., Utrecht, Netherlands, Pierre Fabre Pharma, Alschwil, Switzerland, Roche Diagnostics International Ltd., Reinach, Switzerland, Roche Pharma AG, Reinach, Switzerland, Sarstedt AG \& Co., Sevelen, Switzerland and Nümbrecht, Germany, Schering-Plough International, Inc., Kenilworth, New Jersey, USA, Tem International GmbH, Munich, Germany, Verum Diagnostica GmbH, Munich, Germany, Vifor Pharma, Munich, Germany, Vienna, Austria and Villars-sur-Glâne, Switzerland, Vifor (International) AG, St. Gallen, Switzerland. $\mathrm{PB}$ received honorary for lecture from LFB and Octapharma. MM received travel support, lecture and advisory board fees and research support from Astra Zeneca, Bayer, Biotest, CSL Behring, TEM International/ILWerfen and LFB Biomedicaments.

JSD received lecture fees from LFB Laboratoires France.

OG received research funding from Bayer Healthcare, Boehringer Ingelheim, Novo Nordisk, Biotest, CSL Behring and Nycomed and honoraria for lectures and consultancy support from Bayer Healthcare, Boehringer Ingelheim, CSL Behring, Octapharma, Sanofi, Pfizer and Portola.

The other authors declare that they have no competing interests. 


\section{Author details}

'Department of Anaesthesiology, Aarhus University Hospital, Aarhus, Denmark. ${ }^{2}$ Department for General and Surgical Critical Care Medicine, Medical University Innsbruck, Innsbruck, Austria. ${ }^{3}$ Department of Anesthesiology and Critical Care Medicine, Lyon Sud Hospital and Claude Bernard University, Lyon Est School of Medicine, Lyon, France. ${ }^{4}$ Grenoble Alps Trauma Center, Department of Anesthesiology and Intensive Care Medicine, Grenoble University Hospital, Grenoble, France. ${ }^{5}$ Weill-Cornell Medicine-Qatar, Department of Anesthesiology, ICU \& Perioperative Medicine, Hamad Medical Corporation, Doha, Qatar. ${ }^{6}$ Department of Anesthesiology, RWTH Aachen University Hospital, Aachen, Germany. ${ }^{7}$ Institute of Anesthesiology, University and University Hospital of Zurich, Zurich, Switzerland. ${ }^{8}$ Department of Anesthesiology and Intensive Care Medicine, AUVA Trauma Centre Salzburg, Academic Teaching Hospital of the Paracelsus Medical University, Salzburg, Austria. ${ }^{9}$ Ludwig Boltzmann Institute for Experimental and Clinical Traumatology, Vienna, Austria. ${ }^{10}$ Department of Traumatology and Orthopedic Surgery, Cologne-Merheim Medical Center, Institute for Research in Operative Medicine (IFOM), University Witten-Herdecke, Cologne, Germany.

Received: 6 June 2019 Accepted: 21 June 2019

Published online: 01 July 2019

\section{References}

1. Sperry JL, Guyette FX, Brown JB, Yazer MH, Triulzi DJ, Early-Young BJ, Adams PW, Daley BJ, Miller RS, Harbrecht BG, Claridge JA, Phelan HA, Witham WR, Putnam AT, Duane TM, Alarcon LH, Callaway CW, Zuckerbraun BS, Neal MD, Rosengart MR, Forsythe RM, Billiar TR, Yealy DM, Peitzman AB, Zenati MS, PAMPer Study Group. Prehospital plasma during airmedical transportation of trauma patients at risk for hemorrhagic shock. N Engl J Med. 2018;379:315-26.

2. Moore HB, Moore EE, Chapman MP, McVaney K, Bryskiewicz G, Blechar R, Chin T, Burlew CC, Pieracci F, West FB, Fleming CD, Ghasabyan A, Chandler J, Silliman CC, Banerjee A, Sauaia A. Plasmafirst resuscitation to treat haemorrhagic shock during emergency ground transportation in an urban area: a randomised trial. Lancet. 2018:392:283-91.

3. Innerhofer $P$, Fries $D$, Mittermayr $M$, Innerhofer $N$, von Langen $D$, Hell $T$, Gruber G, Schmid S, Friesenecker B, Lorenz IH, Ströhle M, Rastner V, Trübsbach S, Raab H, Treml B, Wally D, Treichl B, Mayr A, Kranewitter C, Oswald E. Reversal of trauma-induced coagulopathy using first-line coagulation factor concentrates of fresh frozen plasma (RETIC): a singlecenter, parallel-group, open-label, randomised trial. Lancet Haematol. 2017;4(6)::258-71.

4. Wutzler S, Maegele M, Wafaisade A, Wyen H, Marzi I, Lefering R. Risk stratification in trauma and haemorrhagic shock: scoring systems derived from the TraumaRegister DGU( $\left.{ }^{(}\right)$. Injury. 2014;45:S29-34.

5. Dunbar NM, Chandler WL. Thrombin generation in trauma patients. Transfusion. 2009:49:2652-60.

6. Holland LL, Foster TM, Marlar RA, Brooks JP. Fresh frozen plasma is ineffective for correcting minimally elevated international normalized ratios. Transfusion. 2005;45:1234-45.

7. Cap AP, Pidcoke HF, Spinella P, Strandenes G, Borgman MA, Schreiber M, Holcomb J, Tien HC, Beckett AN, Doughty H, Woolley T, Rappold J, Ward K, Reade M, Prat N, Ausset S, Kheirabadi B, Benov A, Griffin EP, Corley JB, Simon CD, Fahie R, Jenkins D, Eastridge BJ, Stockinger Z. Damage control resuscitation. Mil Med. 2018;183(Suppl 2):36-43.

8. Floccard B, Rugeri L, Faure A, Saint Denis M, Boyle EM, Peguet O, Levrat A, Guillaume C, Marcotte G, Vulliez A, Hautin E, David JS, Negrier C, Allaouchiche B. Early coagulopathy in trauma patients: an on-scene and hospital admission study. Injury. 2012;43:26-32.

9. Spahn DR, Bouillon B, Cerny V, Duranteau J, Filipescu D, Hunt BJ, Komadina R, Maegele M, Nardi G, Riddez L, Samama CM, Vincent JL, Rossaint R. The European guideline on management of major bleeding and coagulopathy following trauma: fifth edition. Crit Care. 2019;23(1):98.

10. Wu F, Kozar R. Fibrinogen protects against barrier dysfunction through maintaining cell surface syndecan-1 in vitro. Shock. 2019; 51(6):740-4.

11. Juffermans NP, Wirtz MR, Balvers $K$, Baksaas-Aasen $K$, van Dieren $S$, Gaarder C, Naess PA, Stanworth S, Johannson PI, Stensballe J, Maegele M, Goslings JC, Brohi K, the TACTIC partners. Towards patient-specific management of trauma hemorrhage: the effect of resuscitation therapy on parameters of thromboelastometry. J Thromb Haemost. 2019;17:441-8.

\section{Publisher's Note}

Springer Nature remains neutral with regard to jurisdictional claims in published maps and institutional affiliations. 